\title{
From Flat Membranes to Fabrication of Hollow Spun Fibers of Polyethersulfone/ Polyvinylpyrillidone (PES/PVP) - Correlative Scanning Electron Microscopy (FE- SEM), 3-D X ray Microscopy and Ultrafiltration Properties
}

Pooja Bajaj ${ }^{1}$, Albin Berzinis ${ }^{1}$, Rachel Halbfinger, Carl Strom ${ }^{1}$, Lars Peters ${ }^{2}$, Matthias Wessling ${ }^{2}$, Naomi Kotwal $^{3}$

${ }^{1}$ Sabic, 1 Noryl Avenue, Selkirk, New York 12158 USA, ${ }^{2}$ RWTH Aachen University, Aachen, Germany, ${ }^{3}$ Carl Zeiss X-ray Microscopy Inc. USA

In a previous report, we presented the application of low voltage field emission scanning electron microscopy to characterize the structure and morphology of lab scale flat membranes cast using Polyethersulfone/polyvinylpyrrolidone (PES/PVP) (Figure 1a, b) [1 and all references within]. Morphology was insightful in tuning the compositional variables and optimizing the design space for translation into hollow spun fibers. In this work, morphological comparison of flat membranes with the hollow spun fibers and their performance properties will be presented. For (PES/PVP) based hollow fiber spinning, the dope solution containing PES (BASF E6020P) at 14 weight\% and polyvinylpyrrolidone (PVP) K90 at 2 weight \%, PVP K30 at 5 weight \%, were blended into a homogenous solution. The blending of low and high K-value PVP is shown to be important in controlling the surface pore structure and tendency towards macro void formation using phase inversion process from n-methylpyrrolidone (NMP) into water [2]. Fiber spinning was performed by dry-wet immersion precipitation using a bore solution of $70 \mathrm{wt} \%$ deionized water and $30 \mathrm{wt} \% \mathrm{NMP}$ [2]. Dope solution along with the bore liquid were simultaneously pumped through a double orifice spinneret and after passing the air gap, immersed into the water coagulation bath. Figure 1c visualizes the fiber spinning process. The fibers were post treated by washing in $70^{\circ} \mathrm{C}$ pure water for $3 \mathrm{~h}$ and air dried. Some fibers were immersed for 24 into a mixture of water/glycerol $(80 \mathrm{wt} \% / 20 \mathrm{wt} \%)$ prior to the drying step. Lab scale hollow fiber membrane modules were prepared from these as spun and dried, and glycerol post treated and dried fibers and tested for the clean water flux and molecular weight cut off measurements. Non-destructive 3-D pore distribution was visualized using Carl Zeiss Xradia 510 Versa and Xradia 810 Ultra $X$ ray microscopy using Zernike phase contrast mechanism to benefit for the otherwise low attenuating membrane material [3].

FESEM characterization of hollow spun fibers of PES/PVP reveal complimentary morphology as seen for flat membranes. Figure 1d shows a low magnification cross section morphology of a hollow fiber membrane with an open, interconnected pore structure highlighting a dense nanoporous skin layer, nano-to-microporous sublayer and macro voids which penetrate the outer membrane wall (Figure1e). Interestingly, the dense inner membrane surface shows pore collapse on as-dried fibers (Figure 1f) that could be reversed when fibers were immersed in glycerol/water prior to drying (Figure 1g). The high resolution images highlight the effect of glycerol in revealing the open nano channels on the selective layer, oriented in the fiber draw direction (Figure 1g). This surface morphology findings of the hollow fibers provide visual understanding and correlation on failing fibers (as spun and dried) compared to functional performance of fibers (glycerol treated and dried) as monitored using clean water flux and molecular weight cut off experiments (Table 1). X ray microscopy (XRM) reveals complimentary pore distribution in 3-D as seen for the cross sectional morphology for flat membranes (figure $2 \mathrm{a}, \mathrm{b}, \mathrm{c}, \mathrm{d}$ ). The cross section was visualized at $65 \mathrm{~nm}$ voxel resolutions using Xradia 510 Versa (Figure $2 \mathrm{e}, \mathrm{g}, \mathrm{h}$ ). The top dense layer was then scanned at high resolution of $32 \mathrm{~nm}$ voxel to resolve less than $100 \mathrm{~nm}$ features (Figure 2 f).In summary, combining complimentary techniques opens the opportunity to not 
only understand the composition variables, but also understand the spinning process attributes that can be tweaked towards robust material characterization and promote new product developments. Future work is focused on computing quantitative metrics based on 3D (e.g. porosity, pore size distribution, etc.) and combining for 4D dynamic (in-situ) studies.

\section{References:}

[1] P. Bajaj et al, Microsc. Microanal. 20 (2014), p. 2100.

[2] L. Gambro et al, (2013), PCT/EP2013/054263 WO2013/131848A1

[3] A. P. Merkle and J. Gelb, Microsc. Today, 21 (2013), p. 10.
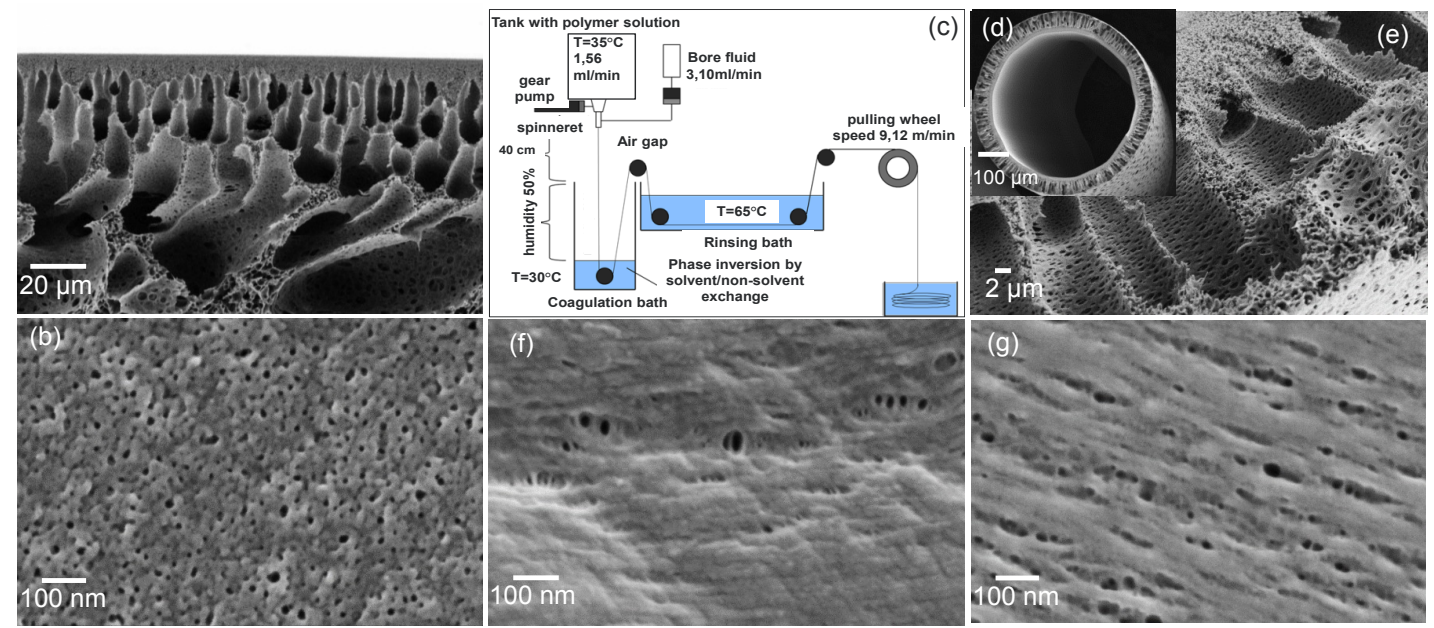

Figure 1. (a) SEM cross sectional and (b) surface morphology of flat membranes of PES/PVP. (c) Fiber spinning process to generate hollow membrane fibers of PES/PVP. (d), (e) SEM cross-sectional and (f) surface morphology of as spun hollow fiber membrane of PES/PVP (g) role of additive, glycerol on opening the porosity of the inner selective layer of the PES/PVP hollow fiber.

Table 1. Performance properties of hollow spun fibers of PES/PVP, as spun and dried and after immersion in glycerol before drying.

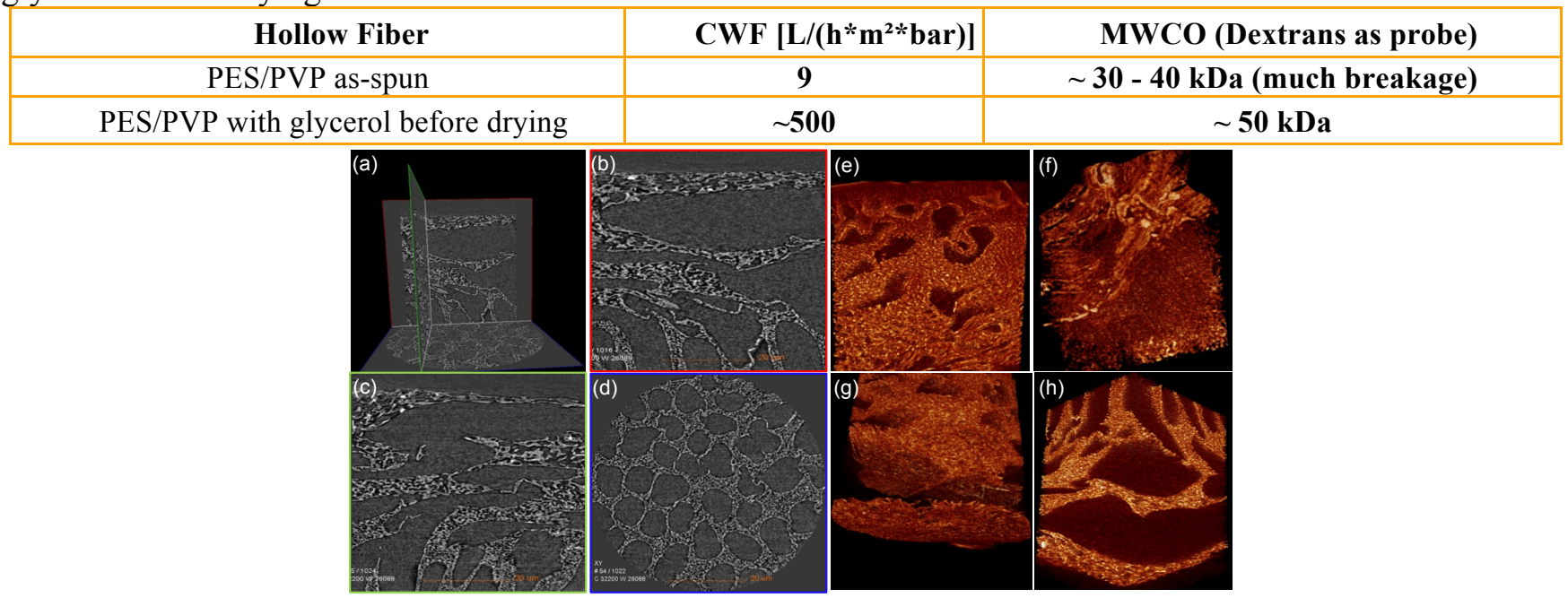

Figure 2. (a) $X$ ray microscopy (XRM) based 3-D viewer. (b), (c), (d) virtual cross sections taken at the orthogonal planes XY, YZ and XZ. (e),(g), (h) High resolution 3D rendered volume that can be combined for further segmentation, thresholding, binarization and quantitative analysis for 3-D porosity and interconnectedness of pore channels at $65 \mathrm{~nm}$ voxel resolution using XRM Versa 510 and (f) $32 \mathrm{~nm}$ voxel resolution using XRM Ultra 810. 